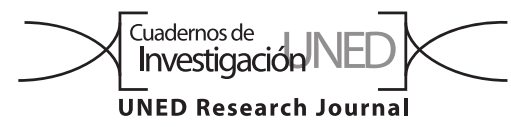

\title{
Nuevos desafíos para la educación ambiental: la vulnerabilidad y la resiliencia social ante el cambio climático
}

\author{
Édgar J. González Gaudiano, Laura Bello Benavides, Ana Lucía Maldonado González, Gloria Elena Cruz Sánchez \\ \& Luis Mario Méndez Andrade
}

Instituto de Investigaciones en Educación, Universidad Veracruzana, Xalapa, Veracruz, México, egonzalezgaudiano@gmail.com, laura_bello310@hotmail.com, anmaldonado@uv.mx, gloriaelena_cruz5@hotmail.com, luis_mma@hotmail.com

Recibido 03-VII-2018 • Corregido 15-XII-2018 • Aceptado 14-I-2019

\begin{abstract}
New challenges for environmental education: vulnerability and social resilience in the face of climate change. The state of Veracruz, Mexico, has suffered frequent flooding in recent years linked to climate change. The aim of this study was to identify the vulnerability and social resilience in school youth at three locations: Cotaxtla, Tlacotalpan y José Cardel. A survey was applied to 411 students and interviews were carried out with 16 key stakeholders. The findings of this consultation process indicated a lack of knowledge in the actions to manage their vulnerability and the regional impacts aggravated by climate change, which was used as an input to design environmental education workshops on the vulnerability and social resilience to climate change. A total of 436 students and 42 teachers participated in the workshops, with involvement from government agencies. This process achieved a greater sensibilization of the students and teachers to the regional impacts of climate change, mitigation and adaptation actions, inter and intracommunity networks were developed and risk maps were created. The work carried out is the beginning in a series of environmental education tasks that need to be developed, with the aim that the communities, through their social learning, can strengthen and energize their social resilience in the face of the risks of climate change.
\end{abstract}

Key words: Veracruz, Mexico, coastal zones, flooding, risk management, environmental education.
RESUMEN: El estado de Veracruz, México, ha sufrido en años recientes inundaciones frecuentes asociadas al cambio climático. El propósito de este estudio fue conocer la vulnerabilidad y resiliencia social en jóvenes de bachillerato de tres localidades: Cotaxtla, Tlacotalpan y José Cardel. Se aplicó un cuestionario a 411 estudiantes y se realizaron 16 entrevistas a actores clave. Los resultados de dicha consulta indicaron desconocimiento de acciones para gestionar su vulnerabilidad y de los impactos regionales agravados por el cambio climático, lo cual se utilizó como insumo para el diseño de talleres de educación ambiental sobre la vulnerabilidad y resiliencia social ante el cambio climático. En los talleres participaron un total de 436 estudiantes y 42 profesores de bachillerato, con participación de instancias gubernamentales. Por medio de este proceso se logró una mayor sensibilización de los estudiantes y profesores sobre los impactos regionales del cambio climático, y de acciones de mitigación y adaptación, se conformaron redes inter e intracomunitarias y se elaboraron mapas de riesgo. El trabajo realizado es el inicio de una serie de tareas de educación ambiental que se han de continuar trabajando, a fin de que las comunidades, a partir de sus aprendizajes sociales, dinamicen y fortalezcan su resiliencia social frente a los riesgos del cambio climático.

Palabras clave: Veracruz, México, zonas costeras, inundaciones, gestión de riesgo, educación ambiental.
El cambio climático se ha posicionado como el gran problema y desafío mundial del siglo XXI (González Gaudiano, 2012). Es un fenómeno complejo que en años recientes ha aglutinado múltiples enfoques. Uno es el económico dados los crecientes costos en materia de adaptación a cambios inminentes y de mitigación de gases de efecto invernadero, así como por la necesaria transformación del modelo energético dominante. Otro es el ecológico, por sus severas implicaciones en la biodiversidad, los océanos, la desertificación, entre otros, y sus efectos en el sistema alimentario. Además, hay un enfoque político, ante la imperiosa urgencia de coordinar esfuerzos y compromisos internacionales para encarar un fenómeno global. Sumado a un enfoque social, por afectaciones en la calidad de vida de la población humana (IPCC, 2014). La educación ambiental no escapa a tal participación.

El cambio climático ha capturado la atención de los medios de comunicación, incrementando la preocupación social; ha empezado a elevarse como prioridad en 
el entorno científico, económico y político. Si bien toda la población mundial ya es y será aún más afectada por el fenómeno, existen sectores que por sus condiciones de vulnerabilidad física, social y motivacional-actitudinal (Anderson \& Woodrow, 1989) se ven más impactados. Este es el caso de la población del estado de Veracruz, México, especialmente la que habita en zonas costeras. Dada la presencia de fenómenos hidrometeorológicos recurrentes potenciados por el cambio climático, estas poblaciones están padeciendo severas consecuencias, como las inundaciones en zonas de la planicie costera veracruzana (Tejeda, 2012).

Aquí es donde se centra el propósito de la presente investigación: explorar las características de la vulnerabilidad social de tres poblaciones del estado de Veracruz frente a los embates del cambio climático, así como la manera de reducirla mediante el desarrollo de capacidades sociales para gestionar riesgos y fortalecer la resiliencia social. Esta tarea la hemos abordado recurriendo a las estrategias de la educación ambiental formal, específicamente en escuelas de bachillerato.

La vulnerabilidad está asociada con el debilitamiento de las relaciones de los componentes de un sistema impactando directamente en el bienestar de los que de él dependen. El concepto ha sido definido desde numerosas disciplinas con base en la presencia de una amenaza (de origen físico o antrópico) vinculada a condiciones objetivas de existencia y determinada representación social del riesgo, a su vez relacionada con las dimensiones cultural e ideológica (Adger, 1999, 2000; Aguirre, 2004; Anderson \& Woodrow 1989).

La vulnerabilidad puede estar referida a las características de una persona, grupo o comunidad y su situación específica; estas inciden en la capacidad de anticipar, lidiar, resistir y recuperarse del impacto de una amenaza (Wisner, Blaikie, Cannon \& Davis, 2004). En el caso de las comunidades, se comprende como un proceso en el que interactúan la amenaza y la vulnerabilidad para generar las condiciones que subyacen a un desastre, atravesadas por cuestiones económicas, ambientales, sociales y políticas (Adger, 2000; Aguirre, 2004). La vulnerabilidad es física cuando involucra aspectos de clima, ubicación e infraestructura de la población, servicios públicos, de salud, de educación y otros (Anderson \& Woodrow, 1989). Es motivacional-actitudinal cuando se relaciona con la autoimagen comunitaria relacionada con las capacidades para enfrentar riesgos. La vulnerabilidad es social cuando tiene que ver con actividades socioeconómicas, las instituciones y el sistema político en el que está inscrita la comunidad.
La vulnerabilidad social de una comunidad se manifiesta cuando experimenta estrés socio-ambiental y se alteran sus medios y condiciones de vida, al estar frente a un evento perturbador, en nuestro caso fenómenos hidrometeorológicos agravados por el cambio climático (Adger, 1999). Para comprender los procesos que inciden en la vulnerabilidad social, en esta investigación la hemos estudiado desde la perspectiva que considera que ésta es un componente de la comunidad y toma en cuenta sus características geoclimáticas, así como sus recursos culturales, sociales y económicos (Aguirre, 2004).

La vulnerabilidad se distingue por ser dinámica, acumulativa, desigual y situada, y es posible desplegar acciones para reducirla (González Gaudiano \& Maldonado González, 2017). Estas acciones estarían orientadas a fortalecer la resiliencia. De acuerdo con Adger (2000) y Cacioppo, Reis y Zautra (2011), la resiliencia social es la capacidad de una comunidad para enfrentar determinadas perturbaciones, desplegando para ello relaciones inter e intracomunitarias pertinentes entre los miembros de la comunidad y actores sociales involucrados, que les permitan además de recuperarse del estrés, generar aprendizajes sociales para transformar su realidad socioambiental. La resiliencia social tiene como rasgo característico poner en el centro de sus tareas las relaciones políticas y de poder entre los actores sociales involucrados, no sólo de los miembros de la comunidad, sino también de aquellos involucrados con la gestión del riesgo y la vulnerabilidad social asociada al mismo, tales como funcionarios gubernamentales, organizaciones civiles, entidades educativas y económicas, principalmente (Keck y Sakdapolrak, 2013). Por lo que el estudio de la resiliencia social se realiza desde la dimensión política para transformar las condiciones socioambientales existentes (Kais \& Isam, 2016).

La resiliencia social se comprende como un proceso dinámico y específico en el que interactúan factores de protección (asociados a formas de organización comunitaria) que se dinamizan frente a determinados factores de riesgo (Kais \& Isam, 2016). Es decir, una comunidad puede ser resiliente frente a los riesgos de inundaciones, pero ello no implica que lo sea frente a otro tipo de riesgos, por ejemplo, desempleo o violencia social. En el estudio de la resiliencia social en nuestra investigación, nos hemos centrado en los factores de protección involucrados frente a los riesgos de las inundaciones.

En ese sentido, una comunidad se distingue por ser compleja, dinámica y generadora de una red de relaciones entre los miembros que la componen (Cheshire, Esparcia \& Shucksmith, 2015; IFRC, 2014), quienes comparten condiciones geoclimáticas, exposición a 
amenazas y riesgos del territorio que habitan, así como los recursos con los que cuentan comunitariamente para desplegar acciones frente a los riesgos que enfrentan (Kais \& Isam, 2016). De aquí que las escuelas desempeñan un papel fundamental porque son centros en los que se desarrollan capacidades para enfrentar riesgos. Aún más, en comunidades rurales y semiurbanas las escuelas suelen funcionar como albergues temporales.

\section{Las localidades de estudio y desarrollo de la investigación}

El propósito de la investigación que aquí presentamos fue, en su primera etapa, conocer la vulnerabilidad y resiliencia social en jóvenes de bachillerato de tres localidades del estado de Veracruz, México (Cotaxtla, Tlacotalpan y José Cardel). Elegimos estas localidades en virtud de que han sufrido en años recientes inundaciones frecuentes. En el cuadro uno presentamos algunas de sus características. Se desarrolló mediante la aplicación de un cuestionario ad hoc, cuya selección de la población fue el criterio de muestra representativa. El tamaño de la muestra fue de 411 estudiantes, con un error admitido de
$3,96 \%, B=0,03965$. Para profundizar en el conocimiento de la vulnerabilidad social se recurrió además a entrevistas a actores clave, en total se realizaron 16 entrevistas (ver González Gaudiano, Maldonado González, Cruz Sánchez, Meza Ortíz \& Méndez Andrade, 2015; González Gaudiano y Maldonado González, 2017; González Gaudiano Maldonado González \& Sánchez Cruz, 2018). La técnica para el estudio de los datos cuantitativos fueron estadísticas descriptivas, utilizando como recurso el software SPSS. Para los datos cualitativos se recurrió a la técnica de análisis de contenido (Bardin, 2002) y se recurrió al software Atlas-ti.

En la segunda etapa de la investigación, con base en el análisis de los datos obtenidos, se buscó generar experiencias de educación ambiental orientadas a fortalecer la resiliencia social. La investigación se realizó durante 2015 y 2017. La edad de los jóvenes de bachillerato con quienes se trabajó osciló entre 15 y 18 años. En la figura 1 se muestra la ruta metodológica de la investigación.

En la figura 1 esquematizamos una parte del trabajo realizado en la segunda etapa del estudio, consistente en el diseño y realización de tres bloques de talleres de educación ambiental, dirigidos a estudiantes y profesores

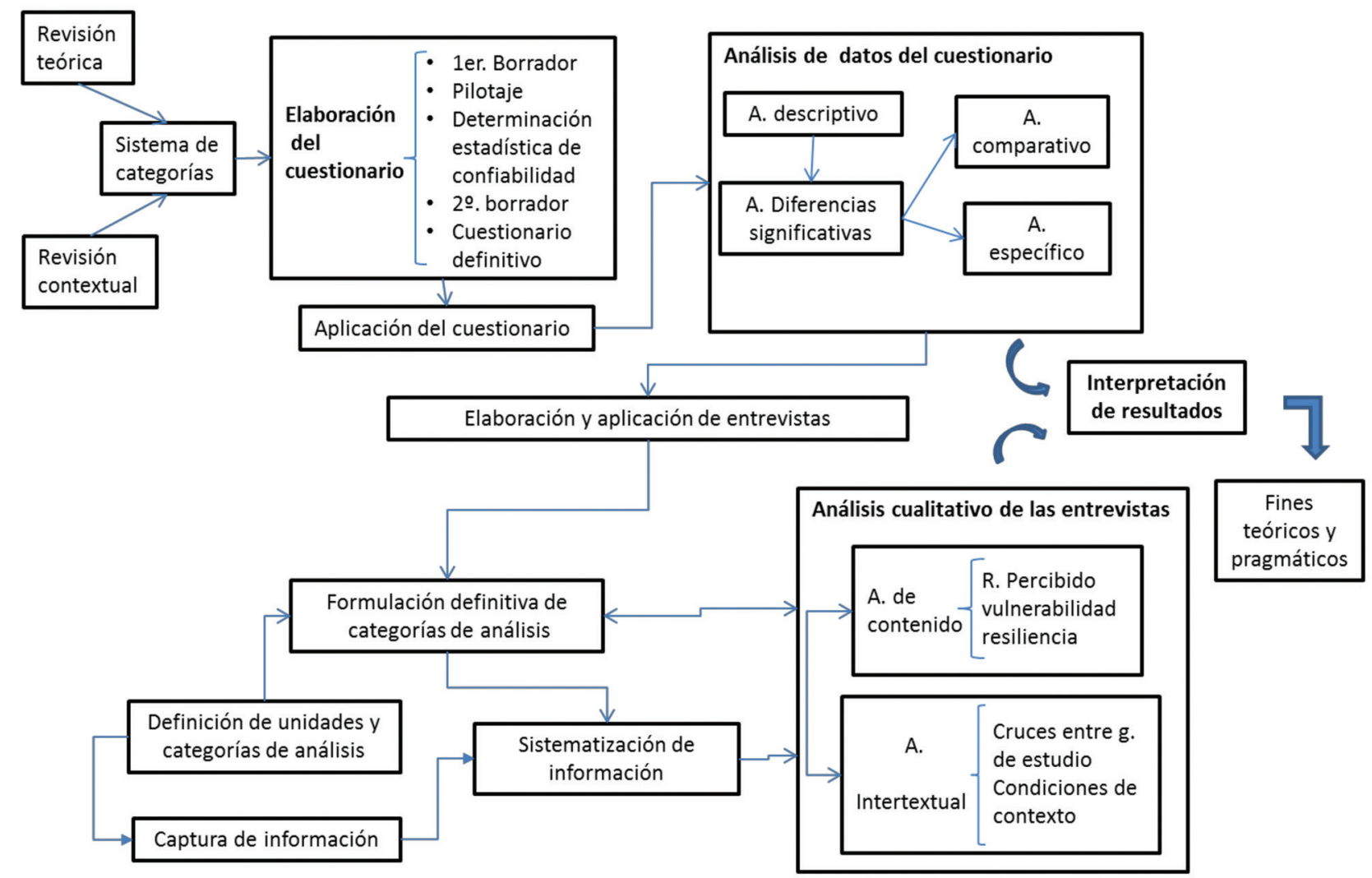

Fig. 1. Ruta metodológica diseñada para la obtención y análisis de la información. 
CUADRO 1

Características geográficas y poblacionales de las comunidades de estudio

\begin{tabular}{lccc}
\multicolumn{1}{c}{ Datos del Municipio } & La Antigua & Tlacotalpan & Cotaxtla \\
Localidad de estudio & José Cardel & Tlacotalpan & Cotaxtla \\
Población de localidad de estudio & 19092 habitantes (Urbana) & 7600 habitantes (Semiurbana) & 1167 habitantes (Rural) \\
Rango de temperatura & $24-26^{\circ} \mathrm{C}$ & $24-28^{\circ} \mathrm{C}$ & $24-26^{\circ} \mathrm{C}$ \\
Rango de precipitación & $1100-1300 \mathrm{~mm}$ & $1400-2100 \mathrm{~mm}$ & $1100-1300 \mathrm{~mm}$ \\
Altitud sobre nivel mar & Entre 10 y $100 \mathrm{~m}$ & Entre 5 y $10 \mathrm{~m}$ & Entre 10 y $200 \mathrm{~m}$ \\
Uso de suelo & Agricultura (34\%) y zona urbana (3\%) & Agricultura (21\%) y zona urbana (1\%) & Agricultura (38\%) \\
Vegetación & Pastizal (34\%), selva (15\%), otro (11\%). & Pastizal (66\%) y tular (7\%) & Pastizal (55\%) y selva (7\%)
\end{tabular}

Fuente: Elaboración propia con datos del Gobierno del Estado de Veracruz (2015a, 2015b, 2015c).

de bachillerato. La información obtenida en la primera etapa del proyecto: desconocimiento de acciones para gestionar su vulnerabilidad y de los impactos regionales agravados por el cambio climático, fue el insumo para el diseño de los talleres a desarrollar (González Gaudiano \& Maldonado González, 2017). El propósito de estos talleres fue contribuir a identificar colectivamente y gestionar acciones de resiliencia social, orientadas a la reducción de la vulnerabilidad social derivada de fenómenos hidrometeorológicos, agravados por el cambio climático. Los dos primeros bloques de talleres se realizaron en las escuelas de cada localidad. El tercer bloque de talleres se realizó en las instalaciones del Instituto de Investigaciones en Educación de la Universidad Veracruzana, Campus Xalapa.

El primer bloque de talleres tuvo como propósito central que los participantes comprendieran el fenómeno del cambio climático, sus causas y consecuencias a nivel global y local, así como la manera en la que nuestras acciones cotidianas lo agravan y también las que pueden incidir en su mitigación y en la adaptación al mismo. El punto de partida en las actividades se centró en temas relacionados con el cambio climático, y los problemas de salud y ambientales derivados. El análisis de los datos recabados en esta primera etapa del proyecto reveló desconocimiento hacia estos temas por parte de los jóvenes, concretamente en lo referido a consecuencias locales como las de la salud (virus de zika, chikungunya y dengue) relacionados con inundaciones. Sin embargo y aunque identifican otros problemas de salud y ambientales en sus localidades, en tal reconocimiento no logran dimensionar sus implicaciones a mediano y largo plazos, ni su relación con el cambio climático. El reconocimiento social de un problema, puede ser el detonante en las tareas de resiliencia social.
La realización de este bloque de talleres fue distribuida en actividades participativas coordinadas por profesores universitarios, académicos expertos en los temas de cambio climático, salud y medio ambiente, junto con los investigadores que trabajamos en este proyecto. En cada una de las actividades se articularon los temas señalados con experiencias lúdicas para desplegar acciones relativas a la adaptación al cambio climático. Participaron aproximadamente 250 estudiantes y 14 profesores divididos en siete grupos, durante los meses de octubre y noviembre de 2015.

En el segundo bloque de talleres intervinieron en promedio 150 estudiantes y 20 profesores, entre mayo de 2016 y febrero de 2017. Aquí el propósito fue que la población estudiantil identificara los componentes de su vulnerabilidad social frente a fenómenos hidrometeorológicos agravados por el cambio climático y cómo gestionarlos a través de acciones de resiliencia social. Asimismo, se contó con participación de investigadores en temas relacionados con cambio climático, vulnerabilidad y resiliencia para la realización de algunas de estas actividades, que incluyeron el análisis de programas de prevención de riesgo por inundaciones, la importancia de la conservación del medio ambiente y el cálculo de la huella ecológica. Con los profesores se trabajó en materia de educación ambiental en las actividades escolares con temas como cambio climático y su incorporación como eje transversal en los programas de estudio, así como la relevancia del docente de bachillerato como promotor de la resiliencia social. También, se expusieron algunos ejemplos para poder trabajar estos temas en la escuela.

El tercer y último bloque de actividades se realizó a través del taller "Acciones comunitarias ante el riesgo de inundaciones". En éste participaron 36 estudiantes y ocho profesores de las escuelas de las tres localidades. 


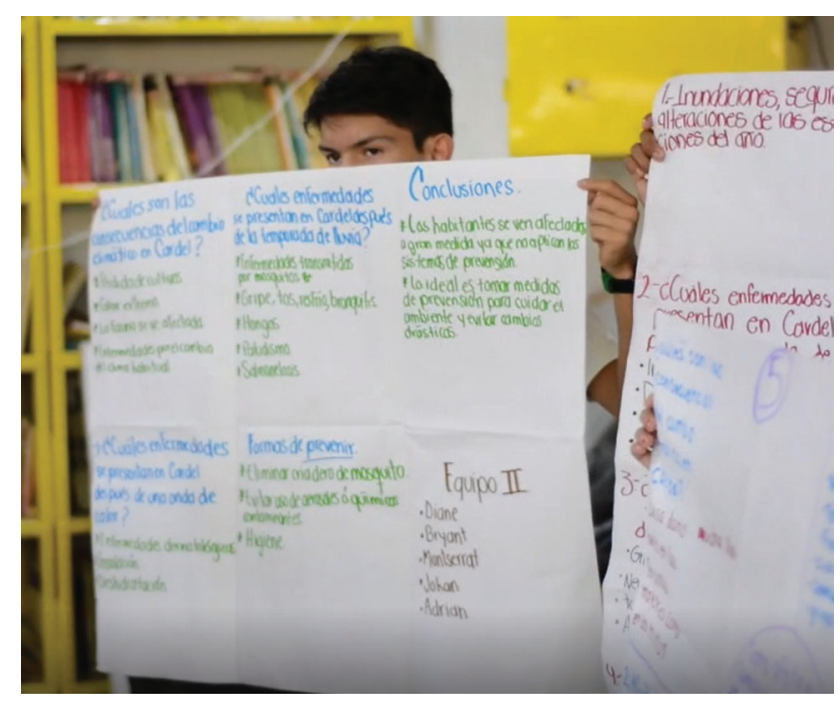

Fig. 2. Reflexiones obtenidas en los talleres participativos.

El propósito fue aportar experiencias de aprendizaje lúdico en estudiantes y profesores, para que desarrollaran sus capacidades de resiliencia, tanto individual como social, en lo relacionado con acciones de respuesta frente al riesgo de inundaciones, antes, durante y después de éstas, a través de sus actividades escolares, con la idea de generar un plan de acciones comunitarias para cada población. Para el desarrollo de este último bloque de talleres se invitó a investigadores expertos en el tema de cambio climático y salud. También se tuvo la participación de representantes de diversas dependencias gubernamentales como las secretarías del Medio Ambiente, de Protección Civil y de Salud del gobierno estatal. Ello con el propósito de tejer redes intracomunitarias de apoyo y cooperación en el tema de inundaciones entre los miembros de las localidades e instituciones gubernamentales. Algunos de los temas abordados por estas instancias gubernamentales fueron: comunicación del cambio climático, vulnerabilidad social (atención a la salud ante inundaciones), resiliencia social y elaboración de mapas de riesgo.

Las actividades se centraron en la identificación de riesgos por impactos hidrometeorológicos. El producto de los talleres fue la elaboración de un mapa de riesgo de cada localidad y la formulación de un plan de acciones a desarrollar desde cada escuela orientadas a la prevención de estos riesgos antes y durante las inundaciones. En la figura 2 se muestran algunas de las reflexiones obtenidas.

Como parte de las estrategias de continuidad y seguimiento a las actividades, los grupos que asistieron a este taller realizado en la ciudad de Xalapa, promovieron actividades resilientes en su escuela de adscripción, una de las cuales fue la reproducción del taller con un mayor número de estudiantes y posteriormente la generación de brigadas juveniles de acción frente a inundaciones. Para ello se entregó a cada grupo un equipo de material didáctico para trabajar en sus escuelas. La duración del taller fue de tres días en jornadas completas de trabajo y fue en el mes de junio de 2017.

En el mes de octubre de 2017 realizamos actividades de cierre del proyecto, a través de grupos focales y entrevistas a estudiantes, con el propósito de valorar cómo a partir de la serie de talleres desarrollados se incidió en las comunidades en la gestión de la resiliencia social en cada una de las comunidades y cómo la escuela había sido partícipe en este proceso. En las tres localidades se identificaron iniciativas por parte de los estudiantes como difusión de mapas de riesgo, organización de charlas sobre los temas de los talleres entre otros grupos de la escuela y conformación de grupos ecologistas para abordar temas ambientales, principalmente.

\section{RESULTADOS}

La escuela desempeñó un rol central, pues al ser ésta espacio social para la gestión de conocimientos y acciones frente a diversos fenómenos que enfrenta la comunidad, uno de éstos las inundaciones, fue el crisol para dinamizar acciones y poder incidir en la comunidad escolar, pero también en las familias a las que pertenecen cada uno de los estudiantes que participaron de estas tareas. Así, se logró influir en un mayor sector de la población en lo relacionado con conocimiento de mapas de riesgo, así como acciones a desplegar antes, durante y después de las inundaciones, además de generar lazos con dependencias gubernamentales a las que pueden acudir.

El primer taller tuvo como resultado un conocimiento más puntual acerca de los impactos regionales del cambio climático, y de acciones de mitigación y adaptación. Se logró una mayor sensibilización cerca de las afectaciones que se reflejó en la solicitud por parte de estudiantes y profesores para dar continuidad a tales actividades. Lo que desembocó en la segunda etapa de talleres participativos, donde el centro de las tareas fue la gestión de la vulnerabilidad. Su identificación y comprensión derivó en propuestas por parte de los jóvenes para conformar brigadas de atención frente a inundaciones, principalmente. Estas inquietudes fueron sistematizadas para concretarse en el tercer taller, donde ya con la participación de instancias gubernamentales se obtuvo como resultado la conformación de redes inter e intracomunitarias y la elaboración de mapas de riesgo. 
Reconocemos que la gestión de la resiliencia social es un proceso dinámico de interacción entre factores de protección y de riesgo; en este sentido, el trabajo que desde la educación ambiental se despliega ha de ser continuo, autocrítico y permanente. Lo que aquí exponemos es el inicio de una serie de tareas de educación ambiental que se han de continuar trabajando, a fin de que las comunidades, a partir de sus aprendizajes sociales, dinamicen y fortalezcan su resiliencia social frente a los riesgos del cambio climático.

\section{REFLEXIONES FINALES}

De lo expuesto se desprenden reflexiones que es importante destacar. La población joven está consciente de la realidad que vive su localidad y muestra interés por participar en actividades de resiliencia social para reducir su vulnerabilidad. Sin embargo, es necesario que desde la educación ambiental se profundice en el estudio de la manera en la que estas poblaciones reconocen y valoran los riesgos que enfrentan, a fin de mejorar el conocimiento de los componentes de su vulnerabilidad social, apoyar en la identificación de su fortalezas y debilidades para incrementar sus factores de protección y gestionar sus factores de riesgo, todo ello en pro de una mayor resiliencia social.

Es en este conjunto de asuntos emergentes donde se ubican los nuevos desafíos de la educación ambiental, no solo en términos de investigación y generación de conocimiento de estos fenómenos centrales, también en los fines pragmáticos de estas investigaciones. Esto es, en la generación de pautas estratégicas tendentes a vincular la vulnerabilidad con el aprendizaje social sobre los temas de referencia, a partir de las condiciones locales que propicien la generación de programas de trabajo conjunto municipal y escolar, dirigidos a la gestión de riesgos derivados de fenómenos climáticos, así como a incrementar la resiliencia social. Pues queda claro que la atención a los desastres es únicamente un paliativo; en tanto la gestión del riesgo en el marco de la resiliencia social es una vía a través de la cual las comunidades pueden actuar individual y colectivamente en términos de adaptación al cambio climático.

Nos queda claro que el desarrollo de la resiliencia social es un proceso donde los talleres realizados son el punto de partida para que estas comunidades gestionen acciones resilientes. Se requiere por lo tanto continuar fortaleciendo las relaciones entre los diferentes actores sociales implicados en la reducción del riesgo frente a los eventos hidrometeorológicos señalados. Esta ha sido la principal dificultad a la que nos hemos enfrentado: en términos económicos para poder financiar la continuación de talleres y gestión de redes de apoyo. También, la inseguridad que actualmente se vive en el estado de Veracruz ha limitado dar seguimiento en la realización de los talleres.

\section{REFERENCIAS}

Adger, W. N. (1999). Social vulnerability to climate change and extremes in coastal Vietnam. World Development, 27(2), 249-269.

Adger, W. N. (2000). Social and ecological resilience: are they related? Progress in Human Geography, Sept(24), 347-364.

Aguirre, B. (2004). Los desastres en Latinoamérica: vulnerabilidad y resistencia. Revista Mexicana de Sociología, 66(3), 485-510.

Anderson, M. B., \& Woodrow, P. J. (1989). Rising from the Ashes. Development Strategies in Times of Disaster. Westview Press-UNESCO, Boulder (Colorado)-París: Westview Press-UNESCO.

Bardin, L. (2002). Análisis de contenido. España: Ed. Akal.

Cacioppo, J., Reis, H., \& Zautra, A. (2011). Social Resilience. The Value of Social Fitness With an Application to the Military. American Psychologist, 66(1), 43-51.

Cheshire, L., Esparcia, J., \& Shucksmith, M. (2015). Community resilience, social capital and territorial governance. Revista de Estudios sobre Población y Desarrollo Rural, 18(enero-julio), 7-38.

Gobierno del Estado de Veracruz (2015a). Cuadernillos municipales, Tlacotalpan. Secretaría de Finanzas y Planeación del Estado de Veracruz, México.

Gobierno del Estado de Veracruz (2015b). Cuadernillos municipales, La Antigua. Secretaría de Finanzas y Planeación del Estado de Veracruz, México.

Gobierno del Estado de Veracruz (2015c). Cuadernillos municipales, Cotaxtla. Secretaría de Finanzas y Planeación del Estado de Veracruz, México.

González Gaudiano, E. (2012). La representación social del cambio climático: Una revisión internacional, Revista Mexicana de Investigación Educativa, 17(55), 1035-1062.

González Gaudiano, E, Maldonado González, A. L., Cruz Sánchez, G. E, Meza Ortíz, S. L., \& Méndez Andrade, L. M. (2015). Novos desafios para a educação ambiental: vulnerabilidade e resiliência social em face dos estragos da mudança climática. Um projeto em municípios de alto risco no estado de Veracruz, México. Rev. Eletrônica Mestrado Educação Ambiental, 32(2), 143-158.

González Gaudiano, E., \& Maldonado González, A.L. (2017). Amenazas y riesgos climáticos en poblaciones vulnerables. El papel de la educación en la resiliencia comunitaria. Teoría Educativa, 29(1), 273-294. 
González Gaudiano, E., Maldonado González, A. L., \& Sánchez Cruz, G. E. (2018) La visión de los jóvenes de bachillerato a su vulnerabilidad y resiliencia social frente a los embates del cambio climático en municipios de alto riesgo a inundaciones. Psyecology, 9(3): 341-364. DOI: 10.1080/21711976.2018.1483568

International Federation of Red Crescent Societies. (2014). Framework for Community Resilience. IFRC: Génova.

IPCC (Grupo Intergubernamental de Expertos sobre el Cambio Climático). (2014). Cambio climático 2014: Impactos, adaptación y vulnerabilidad. Ginebra, Suiza: Organización Meteorológica Mundial.
Kais, S., \& Islam, M. (2016). Community Capitals as Community Resilience to Climate Change: Conceptual Connections. International Journal of Environmental Research and Public Health, 13(12), 1211. MDPI AG. Recuperado de:http://dx.doi.org/10.3390/ijerph13121211.

Tejeda, A. (2012). Las inundaciones de 2010 en Veracruz. Memoria social y medio físico. México, Universidad Veracruzana (Biblioteca del especialista).

Wisner, B., Blaikie, P., Cannon T., \& Davis, I. (2004), At risk: natural hazards, people's vulnerability and disasters, (2a ed.). Londres: Routledge. 